described organizational characteristics critical to implementing Lean and to which they attributed its success so far, including: strong leadership and the importance that leaders embody qualities they are espousing, willingness to engage all levels of staff in the change process, and willingness to adjust performance measures according to new job roles. However, many noted that values and norms surrounding clinical practice are often at odds with the Lean principle of standardizing work to eliminate waste, representing the biggest challenge for physicians who are socialized into a culture where independent thinking and autonomy is valued. The availability of resources was also cited as an important factor in executing changes, including time to do one's regular work while implementing change, time to absorb new ideas and changes, and proper space configurations to support the change. Conclusions: Lean represents a non-traditional approach to managing the delivery of medical care. In a Lean operating system, value is seen first from the patient perspective and while this is a point of easy agreement, how that principle is operationalized can be fraught with challenges that must be negotiated. These challenges may be addressed in part by strong leadership and adequate resources. Further study is currently underway as the effort is extended to additional sites in the organization, with additional findings to be presented on how Lean can be successfully implemented in health care.

Keywords: Lean Management; Implementation Issues; Qualitative Research doi:10.3121/cmr.2013.1176.ps2-13

PS2-14:

Ready to Change? The Role of Employee Engagement, Ownership, and Participation in Managing Change

Dorothy Hung ${ }^{1}$; Eric Wong ${ }^{1}$; Katie Anderson ${ }^{1}$; James Hereford ${ }^{1}$

${ }^{1}$ Palo Alto Medical Foundation for Healthcare, Research and Education

Background/Aims: Healthcare organizations are implementing an array of changes to provide more affordable, high quality care. Many organizations achieve only partial success when implementing improvement initiatives, with half of all failures due to a lack of readiness to change among personnel. This study examines factors that contribute to change readiness among employees in an organization undergoing system-wide transformation. Methods: Baseline data were collected from 706 physicians and staff in 19 primary care departments and 3 call centers in a large ambulatory care system. A validated, multi-dimensional Organizational Change Recipients' Beliefs Scale was used to assess readiness to change. Multivariate regression was used to examine predictors of change readiness among physicians and non-physician personnel. Results: Non-physicians (nurses, medical assistants, administrative staff) reporting a high level of engagement (e.g., "My ideas and suggestions are valued by my department") and ownership (e.g., "I am willing to put in a great deal of effort to help my department succeed") scored significantly high on four dimensions of readiness to change, including perceived appropriateness of the change, anticipated benefit from changes, perceived support for change among peers and leaders, and capability to implement changes $(P<0.01)$. Among physicians, commitment was positively associated with these four dimensions as well as a fifth dimension of readiness -- perceived need for change. Both physicians and non-physicians reporting burnout in the form of emotional exhaustion perceived greater need for change $(P<0.01)$, while those with longer tenure in their department perceived less need for change $(P<0.01)$. Burnout among non-physicians in the form of depersonalizing patients was associated with less perceived capability of implementing changes $(P<0.05)$. Last, participation in decision-making within departments was positively associated with non-physician engagement and ownership $(P<0.05)$ Conclusions: Perceptions about work environment can affect employees' beliefs about changes being undertaken by their organization. Both employee engagement and ownership were instrumental in preparing non-physicians for change; only ownership affected physicians' readiness to change. Burnout and tenure universally affected a perceived need for change. Encouraging staff participation in decision-making can help instill a sense of engagement and ownership among non-physicians, though alternative mechanisms must be sought for preparing physicians for change.

Keywords: Readiness to Change; Employee Engagement/Ownership; Participation in Decision Making

doi:10.3121/cmr.2013.1176.ps2-14
PS2-17:

\section{Does Better Care Cost More?}

\section{Su-Ying Liang ${ }^{1}$; Sukyung Chung ${ }^{1}$; Harold Luft ${ }^{1}$}

${ }^{1}$ Palo Alto Medical Foundation for Healthcare, Research and Education

Background/Aims: Understanding variation in resource use among primary care physicians (PCPs) and its impact on clinical quality and patient outcomes is critical for designing policies to encourage efficiency in delivery without sacrificing quality. This study examined the relationship between PCPs' use of resources, and clinical quality and patient-assessed quality indicators. Methods: We used a dataset linking electronic health records, administrative claims, and patient satisfaction surveys from a large ambulatory group practice with mixed sources of payment. We studied PCPs practicing family or general internal medicine in $2010(n=208)$. For the measure of resource use, we report fee-weighted differences for ambulatory services relative to the overall average ("costs"). For the measures of clinical quality, we combined various indicators pertinent to primary care practice and created a composite average score (number of patients who met the target/number of eligible patients). For patient-rated care quality, we examined the percent of patients who indicated in the survey that they were "very likely" (5 on 1-5 scale) to recommend their provider to others. We tested whether costs differ between PCPs who have superior quality and satisfaction scores and those who have less exceptional scores. Based on tertiles of satisfaction and quality scores, PCPs were classified into three subsets: (1) high-satisfaction/high-quality (HS-HQ), (2) high-satisfaction/ medium-quality (HS-MQ) or medium-satisfaction/high-quality (MS-HQ), and (3) the remainder (non-superior). PCPs with low-satisfaction and lowquality scores were not studied further. Results: Costs were highest in the HS-HQ group $(9.0 \%[ \pm 3.6 \%]$ above the non-superior group, and $8.6 \%[ \pm$ $4.4 \%]$ above the HS-MQ or MS-HQ group). The difference in costs was statistically significant between PCPs with HS-HQ scores and those with non-superior scores $(P=0.01)$. Differences were not significant between PCPs with HS-MQ or MS-HQ scores and those with non-superior scores. Conclusions: These findings are preliminary, as we plan to apply a more detailed risk-adjustment approach. However, the findings suggest that PCPs with both high clinical quality and patient satisfaction scores used approximately $9 \%$ more services for their patients (age and sex adjusted) than PCPs with less exceptional scores.

Keywords: Health Care Utilization; Clinical Quality; PatientAssessed Quality

doi:10.3121/cmr.2013.1176.ps2-17

PS2-18:

Functional Limitations, Home Support, and Responses to Drug Costs Among Medicare Beneficiaries

\section{Christopher Whaley ${ }^{1}$; Mary Reed ${ }^{2}$; John $\mathrm{Hsu}^{3}$; Vicki Fung ${ }^{4}$}

${ }^{1}$ University of California, Berkeley; ${ }^{2}$ Kaiser Permanente Northern California; ${ }^{3}$ Harvard Medical School; ${ }^{4}$ Kaiser Permanente Mid-Atlantic

Background/Aims: Many Medicare beneficiaries have conditions or disabilities that affect their functional status and self-care capabilities. There is limited information on the potential associations between functional limitations, home support, and self-care activities such as managing and taking medications. Methods: We conducted telephone interviews in a stratified random sample of community-dwelling Medicare Advantage beneficiaries in an integrated delivery system, age $65+$ years $(\mathrm{N}=1,201$; response rate $=70.0 \%$ ). Participants reported their functional status as measured by activities of daily living (ADLs; e.g., bathing) and instrumental activities of daily living (IADLs; e.g., preparing meals), and whether they received any support from family members or caretakers in obtaining, paying for, or taking medications. We also examined drug cost-related changes in medication use: cost-reducing behaviors (e.g., switching to generics), costrelated non-adherence (e.g., not refilling), and financial stress (e.g., cutting back on necessities). We used multivariate logistic regression to assess associations between functional status, support with medications, and drug cost responses, adjusting for patient characteristics. Results: Nearly half of respondents $(42 \%)$ reported having a functional limitation: $26.7 \%$ reported $1-2$; and $15.6 \%$ reported $3+$. Among beneficiaries with functional limitations, 